\title{
Non-Surgical Management of Cord Compression in Tuberculosis: A Series of Surprises
}

\author{
Sanganagouda Shivanagouda Patil, Sheetal Mohite, Raghuprasad Varma, \\ Shekhar Y Bhojraj, Abhay Madhusudan Nene \\ Division of Spine Surgery, Department of Orthopaedics, PD Hinduja National Hospital and Medical Research Centre, Mumbai, India
}

\begin{abstract}
Study Design: Prospective study.
Purpose: We present a series of 50 patients with tuberculous cord compression who were offered systematic non-surgical treatment, and thereby, the author proposes that clinico-radiological soft tissue cord compression is not an emergency indication for surgery.

Overview of Literature: Spinal cord compression whether clinical or radiological has usually been believed to be an indication for emergency surgery in spinal tuberculosis.

Methods: Fifty adults were prospectively studied at our clinic for spinal cord compression due to tuberculous spondylitis, between May 1993 and July 2002. The inclusion criteria were cases with clinical and/or radiological evidence of cord compression (documented soft tissue effacement of the cord with complete obliteration of the thecal sac at that level on magnetic resonance imaging scan). Exclusion criteria were lesions below the conus level, presence of bony compression, severe or progressive neurological deficit $/<$ than Frankel grade C) and children below the age of maturity. All patients were treated with a fixed, methodically applied non-surgical protocol including hospital admission, antitubercular medications, baseline somatosensory evoked potentials and a regular clinicoradiological follow-up.

Results: At the time of presentation, 10 patients had a motor deficit, 18 had clinically detectable hyper-reflexia and 22 had normal neurology. Forty-seven of the 50 patients responded completely to non-operative treatment and healed with no residual neurological deficit. Three patients with progressive neurological deficit while on treatment were operated on with eventual excellent recovery. Conclusions: Radiological evidence of cord compression and early neurological signs need not be an emergency surgical indication in the management of spinal tuberculosis.
\end{abstract}

Keywords: Non-surgical management; Spinal tuberculosis; Spinal cord compression

\section{Introduction}

Indications for surgery in spinal tuberculosis (TB) have been widely debated over the years [1-15]. The indications for urgent surgical intervention in spinal TB vary from neurologic deficit due to spinal cord compression (clinical or radiological) [16] to spinal instability. In developing countries, there is a gross mismatch between the disease burden and the surgical facilities that are available. With the literature supporting good neurological outcomes following treatment (medical or surgical) in patients of spinal TB, some of these patients can be

Received Jun 6, 2013; Revised Jul 17, 2013; Accepted Jul 23, 2013

Corresponding author: Sanganagouda Shivanagouda Patil

Division of Spine Surgery, Department of Orthopaedics, PD Hinduja National Hospital and Medical Research Centre,

Veer Savarkar Marg, Mahim West, Mumbai, Maharashtra 400 016, India

Tel: +91-22-2444-7427, Fax: +91-22-2444-0425, E-mail: sanganpatil9@gmail.com 
considered for non-operative treatment, especially the ones who do well with supervised anti-Koch's therapy (AKT). There is very little in the literature that discusses systematic non-surgical treatment in the situation of cord compression. We present a series of 50 patients with tuberculous cord compression who were treated with a methodical non-surgical treatment.

\section{Materials and Methods}

We reviewed 50 consecutive adult patients (who fit the inclusion criteria) treated in our clinic for spinal cord compression due to tuberculous spondylitis, between May 1993 and July 2002. This was a prospective study, with all enrolled patients being given a fixed non-surgical management protocol. There was no 'control' group, as all patients who suited the inclusion criteria were offered the same protocol of management.

\section{Inclusion criterias}

- Clinico-radiological diagnosis of spinal TB

- Documented cord compression on magnetic resonance imaging (MRI) scan, in the form of soft tissue effacement of the spinal cord or the conus medullaris, with complete obliteration of the thecal sac at that level

- With or without associated clinical evidence of spinal cord compression

All of these patients were not biopsy proven cases, as they belonged to the period when we did not perform biopsy routinely in all cases and empirical treatment was often followed.

\section{Exclusion criterias}

- Lesions below the conus level

- Presence of bony compression

- Severe or progressive neurological deficit of Frankel grade $\mathrm{C}$

- Children below the age of maturity

There were 32 females and 18 males. The age ranged from 17 years to 86 years with an average age of 42 years. The average duration of follow-up was 20 months (range, 12-72 months). Seven of the 50 patients had pure cervical spine lesions, while the other 43 had thoracic spine involvement (including cervico-thoracic and thoracolumbar lesions).
On presentation, all patients were admitted to hospital and each patient was evaluated clinically every day. Baseline plain X-rays of the spine and the chest, routine blood checks and an MRI of the spine (if not already done) were done on admission.

All patients were treated with a pre-set, methodically applied conservative treatment with empirical AKT, under very close supervision. At this point in our practice, it was standard practice to treat TB empirically (as TB was an endemic disease in our country and standard diagnostic techniques like computed tomography [CT] guided biopsy and modern culture and sensitivity methods were not available). This included, anti-TB medications ([isoniazide $5 \mathrm{mg} / \mathrm{kg}$, rifampicine $10 \mathrm{mg} / \mathrm{kg}$, ethambutol 15 $\mathrm{mg} / \mathrm{kg}$, and pyrazinamide $25 \mathrm{mg} / \mathrm{kg}$ ] as first line medication), IV methyl-prednisolone and a regular time-bound clinico-radiological follow-up. Patients with no clinical or laboratory response at 3 months were considered to be resistant to first line AKT and were put on second line medications (kanamycin, ciprofloxacin, sparfloxacin, ethionamide and so forth). The treatment of patients with resistance to first line AKT consisted of using at least three second line drugs of which one was an injectable. At the time of this study, the total duration of our standard empirical treatment was 12 months.

The treatment was monitored and supervised by the chest physicians throughout. Patients were evaluated clinically, as well as by radiology, and blood investigation reports were analyzed for inflammatory markers at presentation by a single observer. Total blood count, erythrocyte sedimentation rate, liver function tests and serum uric acid were done to monitor the response to medications as well as the side effects. Healing was judged clinically as well as radiologically as follows.

Clinically, improvement was defined as a significant improvement in spinal pain/tenderness, paraspinal muscle spasm and eventual predisease activity status with weight gain and no residual instability or neurological deficits. On MRI scan, all the patients had presented with an epidural abscess compressing the spinal cord. Radiologically, improvement was defined as significant regression in the epidural abscess/granulation tissue in the immediate follow-up period and marrow reconversion and fatty reconstitution of the diseased bone at the final follow-up. An MRI scan was done at 0, 3, and 12 months to monitor the radiological response to treatment, i.e., resolution of the epidural soft tissue and pus compressing 
the spinal cord. Follow-up in the series ranged from one to five years, with the average follow-up being 2 years.

All patients were not only informed about the nature and duration of treatment they were to undergo but also the importance of a strict follow-up program, and the warning signs and the possibility of eventual surgery.

\section{Results}

The results of our pre-set, methodically applied, conservative treatment under very close observation were as follows. At the time of presentation, 10 patients had a motor deficit of Frankel grade D, 18 patients had clinically detectable hyper-reflexia, while the other 22 patients had normal neurology (Frankel grade E). Seven patients had been advised to have surgery elsewhere and they were admitted for the same. Four patients required more than 4-drug (first line) AKT based on the clinico-radiological criteria and response to first line AKT. Of these, one of the patients was a culture/biopsy proven drug-resistant TB patient.

On MRI scan, all the patients presented with an epidural abscess compressing the spinal cord. Forty-seven out of 50 patients responded completely to non-operative treatment and healed with no residual neurological (Frankel grade E) deficit. Three patients worsened neurologically to Frankel grade $\mathrm{C}$ while on AKT, and hence, they were operated upon with excellent eventual recovery, progressing to normal neurology (Frankel grade E) in the immediate postoperative period, as well as at the final followup.

\section{Discussion}

With the description of the anterior approach in the 1970s, and subsequently, with the technical revolution in spinal reconstruction and instrumentation, spinal TB was being treated increasingly with surgery till the mid 1990s [17]. The surgical options ranged from simple drainage of pus to radical resection of the tuberculous infected focus/ affected vertebra, followed by reconstruction using bone grafts, cages and stabilization with implants.

However, the treatment of spinal TB seems to have come full circle today, with a better understanding of the microbiology of the disease, as well as the better availability of modern culture and sensitivity methods. Spinal TB today is considered a medical disease in the absence of neurological deficit and gross deformity [18-20].

However, there are no clear cut guidelines in the literature regarding how much neurology is significant to warrant surgery. It is obviously difficult to set a cut off point for neurologic deficits, for the movement from nonsurgical to surgical treatment. In the literature, guidelines vary from offering surgery to patients with any grade of neurologic deficit to performing surgery only for patients with advanced motor deficits. Patients with significant neurologic deficits, i.e., TB paraplegia with epidural pus and granulation tissue compressing the spinal cord (on an MRI scan), have straight forward indications for surgery [21], as the prognosis of recovery is better when compared to other pathological compressive lesions. But in the TB spine, most of the times the disease is evolving, i.e., the development of a neurologic deficit is a gradual process with a set pattern. Hence, there remain a large subgroup of spinal TB patients with radiologic epidural cord compression but with only early neurological signs, i.e., with clumsy gait, hyper-reflexia, clonus and early motor deficits (Frankel grade D and E). It is in this group of patients that a trial of conservative treatment can be considered. In our current study, 47 out of 50 such patients responded well to non-surgical treatment and did not end up with progressive neurological worsening, clearly showing that radiological evidence of cord compression and early neurologic signs need not be an absolute indication for surgery in the management of spinal TB.

There always remains a chance of failure, as well as the fear of a worsening neurologic deficit while on conservative treatment, for which close monitoring of the patient's neurological status is extremely important. For this we did (and do advocate) initial monitoring of these patients as inpatients, with twice daily complete neurological examinations to check for the slightest possible deterioration in motor power. The same regimen is continued for two weeks once the patient's condition stabilizes. Patient education and counseling about the red flags and the possibility of emergent surgery while on treatment remain of paramount importance.

Any worsening in motor power or persistence of the preexisting neurologic status despite the patient being on $\mathrm{AKT}$ and the above mentioned supportive treatment calls for a decision to be made regarding surgical treatment. A worsening neurologic deficit or the development of a major neurologic deficit ( $\leq$ Frankel grade $\mathrm{C}$ ) or progressive paraplegia while on treatment under supervision 
becomes an absolute indication for surgical treatment. Epidural soft tissue swelling consisting of pus, granulation tissue and edema may subside and resolve completely with antitubercular medicines alone as shown in Figs. $1-3$. Forty-seven out of fifty (94\%) patients in our series showed complete resorption and healing of the compressing epidural tissue with chemotherapy alone. In three patients (6\%), further worsening of the neurologic deficit ( $\leq$ than Frankel grade C) despite chemotherapy made us consider surgical decompression (Fig. 4). In these operated patients, the neurologic status improved completely to normal in the immediate postoperative period, which persisted even after stopping the AKT, at the final followup.

In the long term follow-up, conservatively treated $\mathrm{TB}$ spine patients developing a kyphotic deformity and its sequelae, i.e., instability (due to concertina collapse in central body lesions) remain a possible entity [22,23], but as substantially evident now, this possibility becomes relevant only in the growing spine $[24,25]$, and the ad- vantages of conservative treatment abundantly outweigh surgical treatment as shown in the literature [26]. In this series, we had six patients with vertebral collapse due to a central body lesion, of which four patients presented with instability complaints at the start of treatment. These complaints resolved completely with time, after treatment with proper AKT.

The issue of drug resistance is very important while treating spinal TB patients [27], as the incidence of drugresistant TB is on rise [28]. Hence, tissue diagnosis and rapid in vitro culture and sensitivity testing methods such as the automated BACTEC Mycobacterial Growth Indicator Tube 960 TB system gain importance [29]. CT guided biopsies increase the accuracy of needle placement, and hence, also the results [30]. In the event of equivocal results of these tests, one can treat spinal TB patients based on the clinico-radiological grounds.

Of importance is patient education and active participation. The patient's views which may include a preference for non-surgical treatment should be given due
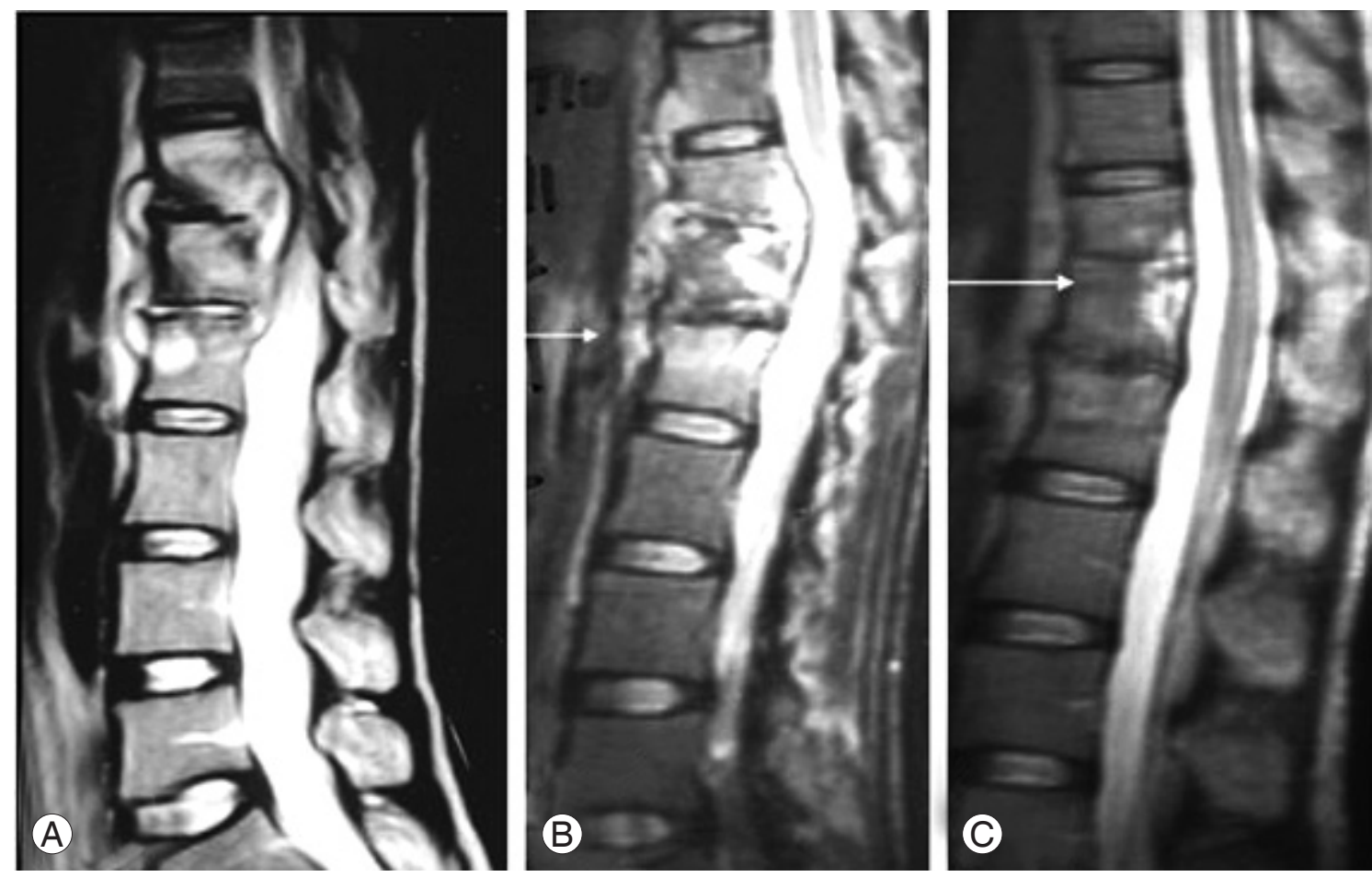

Fig. 1. Magnetic resonance imaging (MRI) scan shows pretreatment (A), during treatment (B) and post-treatment (C) imaging of the thoracolumbar spine of this patient. This patient presented only with early onset neurological signs and tubercular spondylodiscitis of T11-T12 and T12-L1 with epidural pus and granulation tissue compressing the spinal cord and a prevertebral collection lifting the anterior longitudinal ligament (A). The patient showed progressive clinical and radiological improvement with antiKoch's therapy alone (as shown in the MRI at 3 months [B] and 12 months [C]). The MRI scan at the end of treatment shows complete resolution of the spinal cord compression, with good bony fusion of the T11-T12 and T12-L1 and near-normal spinal alignment (C) 

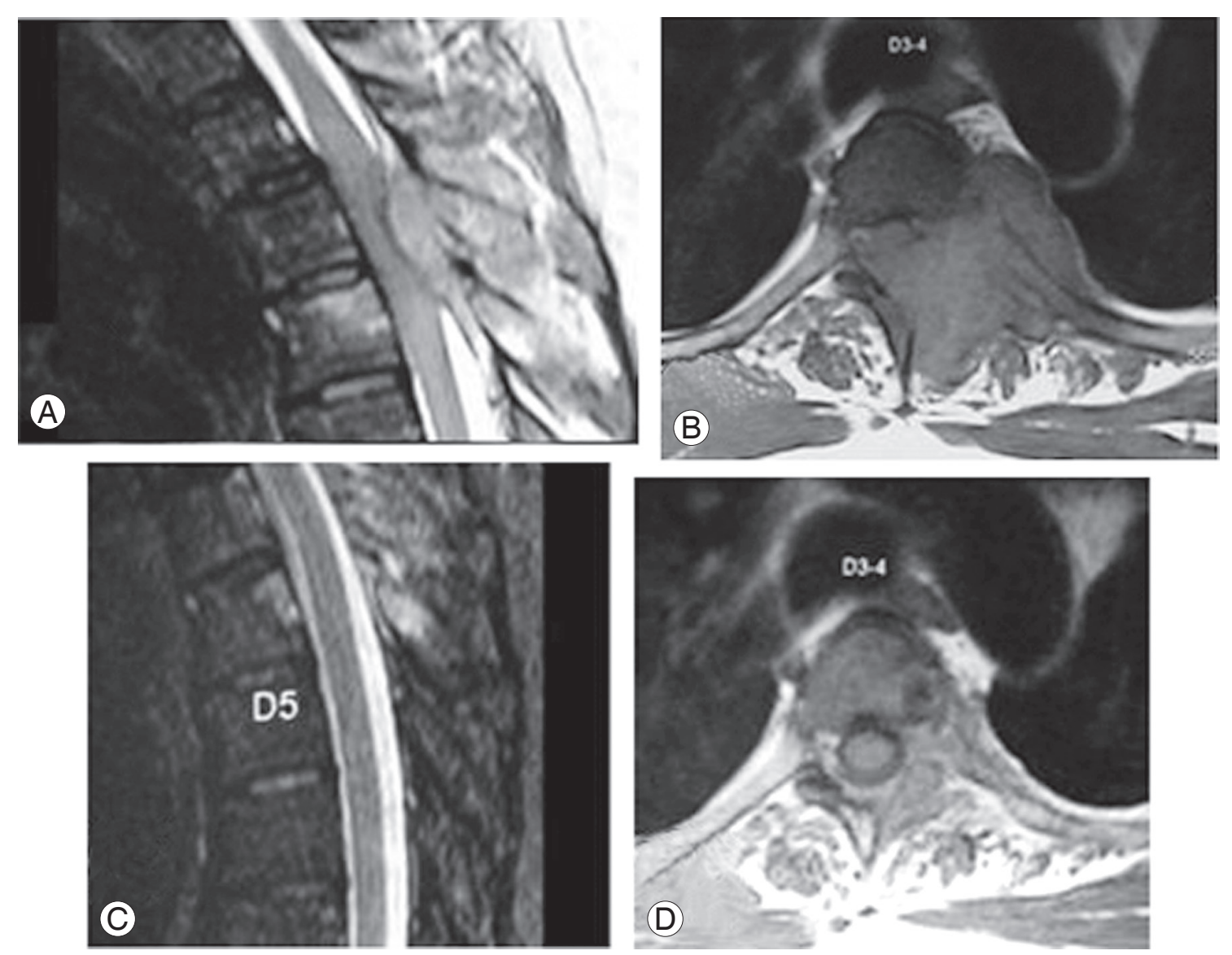

Fig. 2. (A, B) Magnetic resonance imaging scan image of this patient shows posterior element tuberculosis of T3-T4 with a huge posterior epidural pus collection compressing the spinal cord and causing early neurological signs. (C, D) The patient shows complete resolution of the disease, clinically as well as radiologically, with 6 months of conservative treatment.

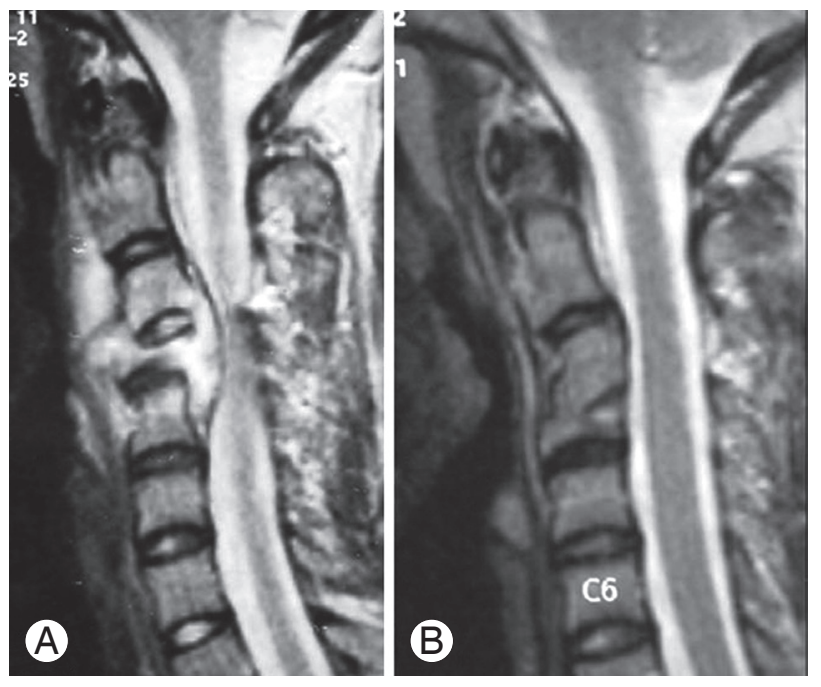

Fig. 3. This figure shows pretreatment $(\mathbf{A})$ and post-treatment $(\mathbf{B})$ magnetic resonance imaging scans of a patient who presented with early neurological signs. The tubercular spondylodiscitis of C3-C4 with concertina collapse of the C4 vertebral body and significant spinal cord compression by pus and granulation tissue (A) completely resolved with 8 months of conservative treatment, with good bony union between C3 and C4 and some loss of segmental lordosis (B). consideration in the presence of spinal cord compression, which otherwise is thought as a surgical problem. The intricate details of the non-operative treatment should be explained to the patient as well as the pros and cons of both types of treatment.

\section{Limitations of the study}

This was a short series of only 50 patients. A larger study group may be required for a more definite conclusion. At the time of this study, the hospital had not adopted biopsy followed by culture and sensitivity testing as a routine policy for each patient before starting AKT. Hence, we really do not know how many of these patients were multi-drug-resistant TB patients and whether their TB would have behaved the same as mycobacterial TB following treatment. A strict follow-up is a must in this kind of study so that worsening neurology can be diagnosed early in order for surgery to be offered at the earliest op- 

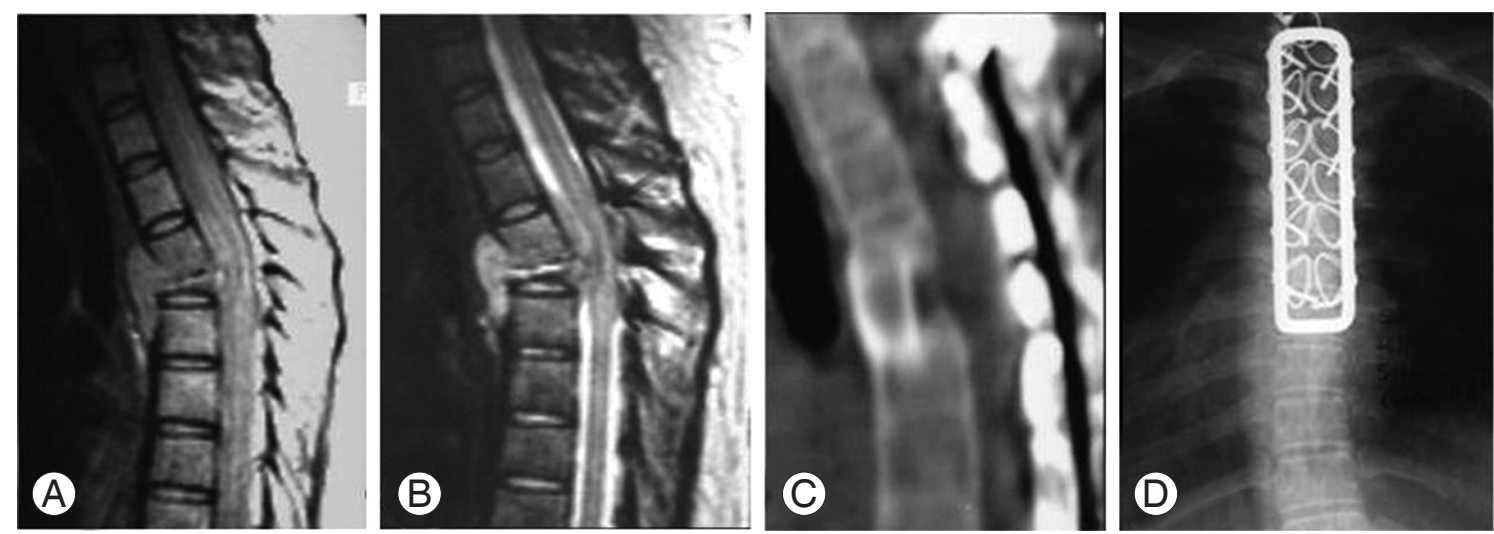

Fig. 4. This patient presented with early neurological signs due to spondylodiscitis of T4-5 with a huge epidural collection of pus and granulation tissue and collapse of the T5 vertebral body causing significant anterior spinal cord compression (A). The patient was put on conservative treatment of anti-Koch's therapy (AKT) hoping for improvement (as seen in this series). But the patient failed to respond to the conservative treatment and presented with a progressive significant neurologic deficit (B) (Frankel C grade). Hence the patient was operated upon and stabilization with a posterior spinal loop rectangle and sublaminar wiring followed by anterior trans-thoracic decompression and bone grafting was done (C). The patient showed excellent eventual neurological recovery in the immediate postoperative period which persisted even after the stopping of AKT (D).

portunity. The follow-up MRI scans at 0, 3, 6 months and before completion of the AKT can be a logistical problem in the case of patients in developing countries. Last but not least, the assessment of neurologic deficits is observer-dependent; hence, inter-observer variability in the assessment of neurologic deficits may lead to the wrong treatment.

\section{Conclusions}

Radiological evidence of cord compression and early neurological signs need not be an emergency surgical indication in the management of spinal TB. Methodically applied non-surgical treatment can certainly give excellent results. However, patient education and participation remain crucial and should be given due importance when implementing non-operative treatment, especially in the presence of spinal cord compression.

\section{Conflict of Interest}

No potential conflict of interest relevant to this article was reported.

\section{References}

1. Albee FH. Transplantation of a portion of the tibia into the spine for Pott's disease: a preliminary report. JAMA 1911;57:885-6.
2. Albee FH. The bone-graft operation for tuberculosis of the spine: twenty years' experience. JAMA 1930; 94:1467-71.

3. Hibbs RA. An operation for Pott's disease of the spine. JAMA 1912;59:433-6.

4. Compere EL, Kleinberg S, Kleiger B, Moore PH, Stewart MJ, Wright PB. Evaluation of streptomycin therapy in a controlled series of ninety cases of skeletal tuberculosis; a public health service cooperative investigation. J Bone Joint Surg Am 1952;34:288-98.

5. Hodgson AR, Stock FE. Anterior spinal fusion a preliminary communication on the radical treatment of Pott's disease and Pott's paraplegia. Br J Surg 1956; 44:266-75.

6. Hodgson AR, Stock FE, Fang HS, Ong GB. Anterior spinal fusion. The operative approach and pathological findings in 412 patients with Pott's disease of the spine. Br J Surg 1960;48:172-8.

7. Smith AD. Tuberculosis of the spine. Results in 70 cases treated at the New York Orthopaedic Hospital from 1945 to 1960. Clin Orthop Relat Res 1968; 58:171-6.

8. Weinberg JA. The surgical excision of psoas abscesses resulting from spinal tuberculosis. J Bone Joint Surg Am 1957;39:17-27.

9. Wilkinson MC. Curettage of tuberculous vertebral disease in the treatment of spinal caries. Proc R Soc Med 1950;43:114-5.

10. Wilkinson MC. The treatment of tuberculosis of the 
spine by evacuation of the paravertebral abscess and curettage of the vertebral bodies. J Bone Joint Surg Br 1955;37:382-91.

11. Friedman B. Chemotherapy of tuberculosis of the spine. J Bone Joint Surg Am 1966;48:451-74.

12. Konstam PG, Blesovsky A. The ambulant treatment of spinal tuberculosis. Br J Surg 1962;50:26-38.

13. Martin NS. Tuberculosis of the spine. A study of the results of treatment during the last twenty-five years. J Bone Joint Surg Br 1970;52:613-28.

14. Moon MS, Kim I, Woo YK, Park YO. Conservative treatment of tuberculosis of the thoracic and lumbar spine in adults and children. Int Orthop 1987;11:31522.

15. Tuli SM. Results of treatment of spinal tuberculosis by "middle-path" regime. J Bone Joint Surg Br 1975; 57:13-23.

16. Nussbaum ES, Rockswold GL, Bergman TA, Erickson DL, Seljeskog EL. Spinal tuberculosis: a diagnostic and management challenge. J Neurosurg 1995;83: 243-7.

17. Rezai AR, Lee M, Cooper PR, Errico TJ, Koslow M. Modern management of spinal tuberculosis. Neurosurgery 1995;36:87-97.

18. Boachie-Adjei O, Squillante RG. Tuberculosis of the spine. Orthop Clin North Am 1996;27:95-103.

19. Moon MS. Tuberculosis of the spine. Controversies and a new challenge. Spine (Phila Pa 1976) 1997;22: 1791-7.

20. Bhojraj S, Nene A. Lumbar and lumbosacral tuberculous spondylodiscitis in adults. Redefining the indications for surgery. J Bone Joint Surg Br 2002;84:5304.

21. Jain AK. Treatment of tuberculosis of the spine with neurologic complications. Clin Orthop Relat Res 2002;(398):75-84.

22. Rajasekaran S. A longitudinal study on the progress of deformity in children with spinal tuberculosis [dissertation]. Chennai: Tamilnadu Dr. MGR University; 1999.

23. Rajasekaran $\mathrm{S}$. The natural history of post-tubercular kyphosis in children. Radiological signs which predict late increase in deformity. J Bone Joint Surg Br 2001;83:954-62.

24. Rajasekaran $\mathrm{S}$. The problem of deformity in spinal tuberculosis. Clin Orthop Relat Res 2002;(398):8592.

25. Wimmer C, Ogon M, Sterzinger W, Landauer F, Stockl B. Conservative treatment of tuberculous spondylitis: a long-term follow-up study. J Spinal Disord 1997;10:417-9.

26. Campos OP. Bone and joint tuberculosis and its treatment. J Bone Joint Surg Am 1955;37:937-66.

27. Pawar UM, Kundnani V, Agashe V, Nene A, Nene A. Multidrug-resistant tuberculosis of the spine--is it the beginning of the end? A study of twenty-five culture proven multidrug-resistant tuberculosis spine patients. Spine (PhilaPa 1976) 2009;34:E806-10.

28. Cohn DL, Bustreo F, Raviglione MC. Drug-resistant tuberculosis: review of the worldwide situation and the WHO/IUATLD Global Surveillance Project. International Union Against Tuberculosis and Lung Disease. Clin Infect Dis 1997;24 Suppl 1:S121-30.

29. Rusch-Gerdes S, Pfyffer GE, Casal M, Chadwick M, Siddiqi S. Multicenter laboratory validation of the BACTEC MGIT 960 technique for testing susceptibilities of Mycobacterium tuberculosis to classical second-line drugs and newer antimicrobials. J Clin Microbiol 2006;44:688-92.

30. Kaltsikis I, Chourmouzi D, Drevelegas K, Potsi S, Moumtzouoglou A, Drevelegas A. Core needle biopsy of spinal lesions under CT guidance: review of 79 cases. J Neurol Surg A Cent Eur Neurosurg 2012; 73:199-203. 